DUKE-TH-96-129

\title{
Thermalization and Lyapunov Exponents in the Yang-Mills-Higgs Theory
}

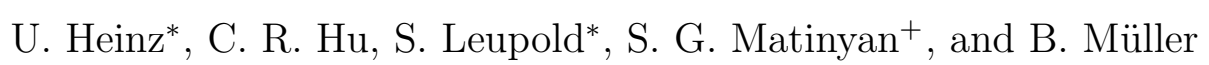 \\ Department of Physics, Duke University, \\ Durham, North Carolina 27708-0305
}

\begin{abstract}
We investigate thermalization processes occurring at different time scales in the Yang-Mills-Higgs system at high temperatures. We determine the largest Lyapunov exponent associated with the gauge fields and verify its relation to the perturbatively calculated damping rate of a static gauge boson.

12.38.Mh, 11.15.Kc
\end{abstract}

Typeset using REVTEX 


\section{INTRODUCTION}

To describe phenomena like heavy ion collisions or the electroweak phase transition in the Early Universe, the methods of quantum field theory must be applied to finite densities and temperatures. The most reliable approach, especially for the case of QCD, are lattice calculations in Euclidean space-time (for a recent overview see e.g. [1]). Unfortunately the results are only applicable to equilibrium processes. Dynamical processes in quantum field theory, on the other hand, are most commonly studied using perturbation theory. In theories with interacting massless degrees of freedom, however, naive perturbation theory in powers of the coupling constant breaks down at high temperature due to infrared divergences caused by the exchange of massless modes. To obtain finite results one must resum classes of Feynman diagrams which lead to dynamical mass generation and thus regulate the infrared singularities. To do this systematically one must disentangle the influence of different momentum scales; in QCD at finite temperature, these are characterized by $T, g T$, and $g^{2} T$ where the last two give the order of magnitude of the dynamical masses for the originally massless electric and magnetic gluonic modes, respectively. An improved one-loop perturbation scheme based on these principles, called hard thermal loop resummation, was developed by Braaten and Pisarski [2] (see also [3] for an overview). Using effective field theory methods, the above separation of the different momentum scales was achieved by Braaten and Nieto for static quantities at high temperature [4].

At high temperature, it is reasonable to expect that phenomena which are dominated by long wavelength modes can be reliably described by classical field theory. This is the basic motivation for the work presented here where we study the classical dynamics of the coupled Yang-Mills-Higgs system. It is well known that the classical non-Abelian gauge field equations are nonintegrable and exhibit dynamical chaos [5]. Therefore one has to rely on numerical methods to study the evolution of classical fields.

The real time evolution of classical $\mathrm{SU}(N)$ Yang-Mills fields on a lattice, for $N=2,3$, was recently studied in Ref. [6]. Starting with randomly chosen initial conditions for the 
gauge fields on each lattice link it was found that the system shows chaotic behavior, i.e. the distance in phase space between two adjacent configurations increases exponentially in time. For systems with sufficiently weak coupling, the time-averaged growth rate (maximal Lyapunov exponent) $\lambda_{\mathrm{N}}$ was shown to scale linearly with the total energy of the system. The slope depends on the number $N$. Even if one starts with highly off-equilibrium configurations, the gauge fields thermalize very fast, i.e. the energy distribution over the lattice soon exhibits a thermal shape. Therefore the total energy of the system can be related to the associated temperature, and the scaling of the maximal Lyapunov exponent with the total energy can be reinterpreted as a scaling with the temperature $T$ - at least in the weak coupling regime. Precisely the relation

$$
\lambda_{\mathrm{N}} a \sim g^{2} T a
$$

which connects dimensionless quantities was established. Here $a$ is the lattice spacing and $g$ the coupling constant. One surmises that the actual value of the proportionality factor

$$
c_{\mathrm{N}}=\frac{\lambda_{\mathrm{N}} a}{g^{2} T a}
$$

survives in the continuum limit $a \rightarrow 0$.

For the classical $\mathrm{SU}(2)$ and $\mathrm{SU}(3)$ gauge field systems, it was found in [6] that the maximal Lyapunov exponent coincides numerically with twice the damping rate $\gamma_{\mathrm{N}}$ of a gauge boson at rest as calculated quantum field theoretically [2] using thermal perturbation theory with hard thermal loop resummation:

$$
\frac{\lambda_{\mathrm{N}} a}{g^{2} T a}=2 \frac{\gamma_{\mathrm{N}}}{g^{2} T}
$$

At first sight it is surprising that the Lyapunov exponent which is deduced from the classical field dynamics by an exact (i.e. non-perturbative) numerical simulation should be related at all with the plasmon damping rate which is calculated perturbatively in quantum field theory. However, the close relation between linear response functions calculated in the hard thermal loop approximation and classical transport phenomena has been known for many 
years [7,8], indicating that the hard thermal loops are essentially classical (see also [9]). In addition it was argued in [10 that the damping of a gluon and the exponential growth of the distance between two adjacent classical trajectories are related. So far there is, however, no rigorous proof for the relation (1.3).

An empirical approach to the question whether the coincidence between the Lyapunov exponent and (twice) the damping rate is only accidental would be to simulate other systems on the lattice and also calculate perturbatively their damping rates. A natural extension of the pure $\mathrm{SU}(N)$ system is to couple it to a matter field. In this article we study the real-time evolution of the coupled SU(2) Yang-Mills-Higgs system. Again we are interested in the thermalization properties and the chaoticity of the fields. To compare with the high temperature results from hard thermal loop calculations we concentrate on the symmetric phase, i.e. we simulate the Higgs behavior in the electroweak plasma above the phase transition in the $\theta_{\mathrm{w}}=0$ limit. Our work was partly motivated by the recent study presented in Ref. [11] in which the question of the connection between the damping rate and the Lyapunov exponent in such a system was also addressed [11]. This paper provides in particular the analytical results for the plasmon damping rates for the gluon and Higgs fields in the hard thermal loop approximation. However, in Ref. [1] the thermalization properties of the classical fields were not taken into account, and thus the determination of the temperature of the system was not dealt with in a satisfactory manner. The correct extraction of the temperature is, of course, crucial to establish relations like (1.1). We postpone a more detailed assessment of the conclusions of Ref. [11] until after presenting our own results.

The organization of the paper is as follows: In Sec. II we formulate the problem. The thermalization properties of the fields are studied in Sec. III. In Sec. IV we compute the Lyapunov exponents, and we discuss our results and compare them with previous work in Sec. V. 


\section{FORMULATION OF THE PROBLEM}

In this Section we present the realization of the coupled SU(2) Yang-Mills-Higgs system on the lattice, describe the initialization of the field variables, discuss the allowed ranges of the relevant parameters in the weak coupling and continuum limits, define the Lyapunov exponents and describe how we measure them.

\section{A. The Yang-Mills-Higgs system on the lattice}

In the temporal gauge $A_{0}^{a}=0$, the Hamiltonian of the coupled SU(2) Yang-Mills-Higgs system in the continuum is given by

$$
H=\int d^{3} x\left[\frac{1}{2} E_{i}^{a} E_{i}^{a}+\frac{1}{2} B_{i}^{a} B_{i}^{a}+\dot{\Phi}^{\dagger} \dot{\Phi}+\left(D_{i} \Phi\right)^{\dagger}\left(D_{i} \Phi\right)+\lambda\left(\Phi^{\dagger} \Phi\right)^{2}\right]
$$

The dot denotes the time derivative, and

$$
\Phi=\left(\begin{array}{c}
\phi_{0}-i \phi_{1} \\
\phi_{2}-i \phi_{3}
\end{array}\right)
$$

is a charged Higgs field in the fundamental representation of $\mathrm{SU}(2)$. The gauge field is

described by vector potentials $A_{i}^{a}(x) . E_{i}^{a}=-\dot{A}_{i}^{a}$ denote the electric fields, $B_{i}^{a}$ the magnetic fields, and $D_{i}$ the covariant derivatives.

As mentioned before, the equations of motion derived from the Hamiltonian (2.1) are not integrable. One has to rely on numerical methods to study the time evolution of the fields. For numerical purposes we discretize the spatial coordinates by using a cubic lattice with $N^{3}$ sites. Time is kept as a continuous variable. For details about the numerical simulation of the real-time evolution of the system we refer to [6].

To realize the coupled SU(2) Yang-Mills-Higgs system on the lattice without losing its gauge symmetry properties we use link variables [12,13

$$
U_{x, i}=\exp \left(-\frac{1}{2} i g a A_{i}^{c}(x) \tau^{c}\right)
$$


instead of vector potentials $A_{i}^{c}$. Here $\tau^{c}$ are the Pauli matrices. The covariant derivative acting on the Higgs field is replaced by the difference

$$
\frac{1}{a}\left(U_{x, i} \Phi_{x+i}-\Phi_{x}\right)
$$

The electric field strength on the lattice is related to the link variables and their time derivatives by

$$
E_{x, i}^{a}=-\frac{i}{g a} \operatorname{tr}\left(\tau^{a} \dot{U}_{x, i} U_{x, i}^{\dagger}\right)
$$

The magnetic energy is expressed in terms of the so-called plaquette operator $U_{x, i j}$, which is the product of all four link variables on an elementary plaquette with sites $(x, x+i, x+$ $i+j, x+j):$

$$
U_{x, i j}=U_{x, i} U_{x+i, j} U_{x+i+j,-i} U_{x+j,-j}
$$

with $U_{x,-i}=U_{x-i, i}^{\dagger}$. The links are directed and hence the plaquettes are oriented. In the continuum limit the plaquette variable $U_{x, i j}$ is related to the local magnetic field $B_{x, k}^{a}$ by

$$
U_{x, i j}=\exp \left(-\frac{1}{2} i g a^{2} \epsilon_{i j k} B_{x, k}^{a} \tau^{a}\right)
$$

It is useful to write the $\mathrm{SU}(2)$ matrices in the form

$$
U=u_{0}-i \tau_{a} u_{a}=\left(\begin{array}{cc}
u_{0}-i u_{3}, & -u_{2}-i u_{1} \\
u_{2}-i u_{1}, & u_{0}+i u_{3}
\end{array}\right)
$$

where the $u_{i}$ are four real numbers, which can be thought of as components of a quaternion. Since $\operatorname{det} U=1$, each link variable $U$ can be expressed in terms of three angles, $\rho_{\mathrm{G}}, \vartheta_{\mathrm{G}}, \varphi_{\mathrm{G}}$, characterizing a three dimensional hypersphere:

$$
U=\cos \frac{\rho_{\mathrm{G}}}{2}-i \hat{\rho}_{\mathrm{G}}^{a} \tau^{a} \sin \frac{\rho_{\mathrm{G}}}{2}
$$

with a three dimensional unit vector

$$
\hat{\rho}_{\mathrm{G}}=\left(\sin \vartheta_{\mathrm{G}} \cos \varphi_{\mathrm{G}}, \sin \vartheta_{\mathrm{G}} \sin \varphi_{\mathrm{G}}, \cos \vartheta_{\mathrm{G}}\right) .
$$


Similarly the four independent components of the Higgs field can be represented as a quaternion:

$$
\Phi=\phi_{0}-i \tau_{a} \phi_{a}=\left(\begin{array}{cc}
\phi_{0}-i \phi_{3}, & -\phi_{2}-i \phi_{1} \\
\phi_{2}-i \phi_{1}, & \phi_{0}+i \phi_{3}
\end{array}\right)
$$

In contrast to the link variable $U$ the Higgs quaternion $\Phi$ has arbitrary length $R$ defined by

$$
R^{2}=\phi_{0}^{2}+\phi_{1}^{2}+\phi_{2}^{2}+\phi_{3}^{2}=\frac{1}{2} \operatorname{tr}\left(\Phi^{\dagger} \Phi\right)
$$

Again we can introduce three angles to get the representation

$$
\Phi=R\left(\cos \frac{\rho_{\mathrm{H}}}{2}-i \hat{\rho}_{\mathrm{H}}^{a} \tau^{a} \sin \frac{\rho_{\mathrm{H}}}{2}\right)
$$

Using the quaternion representations for the link variables and the Higgs fields, the Hamiltonian of the coupled SU(2) Yang-Mills-Higgs system on the lattice reads

$$
H=H_{\mathrm{el}}+H_{\mathrm{mag}}+H_{\mathrm{kin}}+H_{\mathrm{pot}},
$$

where we define the electric energy

$$
H_{\mathrm{el}}=\sum_{x} \epsilon_{\mathrm{el}}(x)
$$

the magnetic energy

$$
H_{\mathrm{mag}}=\sum_{x, i, j} \epsilon_{\mathrm{mag}}(x, i j)
$$

the Higgs kinetic energy

$$
H_{\mathrm{kin}}=\sum_{x} \epsilon_{\mathrm{kin}}(x)
$$

and the Higgs potential energy

$$
H_{\mathrm{pot}}=\sum_{x} \epsilon_{\mathrm{pot}, 2}(x)+\sum_{x} \epsilon_{\mathrm{pot}, 4}(x)+\sum_{x, i} \epsilon_{\mathrm{int}}(x, i),
$$

and the $\epsilon$ 's are energies associated with the respective site, link, or plaquette: 


$$
\begin{aligned}
\epsilon_{\mathrm{el}}(x) & =a^{3} \sum_{i} \frac{1}{2} E_{x, i}^{a} E_{x, i}^{a}, \\
\epsilon_{\mathrm{mag}}(x, i j) & =\frac{4}{g^{2} a}\left[1-\frac{1}{2} \operatorname{tr} U_{x, i j}\right], \\
\epsilon_{\mathrm{kin}}(x) & =a^{3}\left[\frac{1}{2} \operatorname{tr}\left(\dot{\Phi}_{x}^{\dagger} \dot{\Phi}_{x}\right)\right], \\
\epsilon_{\mathrm{pot}, 2}(x) & =6 a\left[\frac{1}{2} \operatorname{tr}\left(\Phi_{x}^{\dagger} \Phi_{x}\right)\right], \\
\epsilon_{\mathrm{pot}, 4}(x) & =a^{3} \lambda\left[\frac{1}{2} \operatorname{tr}\left(\Phi_{x}^{\dagger} \Phi_{x}\right)\right]^{2}, \\
\epsilon_{\mathrm{int}}(x, i) & =-a \operatorname{tr}\left(\Phi_{x}^{\dagger} U_{x, i} \Phi_{x+i}\right) .
\end{aligned}
$$

To derive the last equation we have exploited the unitarity of the link variable $U$ and the fact that the trace of a quaternion is real.

The Hamiltonian (2.14) is scale invariant. To see this we define the dimensionless variables

$$
\bar{t}=t / a, \quad \bar{H}=g^{2} a H, \quad \bar{E}_{x, i}^{a}=g a^{2} E_{x, i}^{a}, \quad \bar{\Phi}_{x}=a g \Phi_{x}, \quad \text { and } \quad \bar{\lambda}=\lambda / g^{2} .
$$

In terms of these we get

$$
\begin{aligned}
\bar{H} & =\sum_{x, i} \frac{1}{2} \bar{E}_{x, i}^{a} \bar{E}_{x, i}^{a}+4 \sum_{x, i, j}\left[1-\frac{1}{2} \operatorname{tr} U_{x, i j}\right] \\
& +\sum_{x} \frac{1}{2} \operatorname{tr}\left(\dot{\bar{\Phi}}_{x}^{\dagger} \dot{\bar{\Phi}}_{x}\right)+\sum_{x, i} \operatorname{tr}\left[\bar{\Phi}_{x}^{\dagger} \bar{\Phi}_{x}-\bar{\Phi}_{x}^{\dagger} U_{x, i} \bar{\Phi}_{x+i}\right]+\bar{\lambda} \sum_{x} \frac{1}{4}\left[\operatorname{tr}\left(\bar{\Phi}_{x}^{\dagger} \bar{\Phi}_{x}\right)\right]^{2}
\end{aligned}
$$

where the dot now denotes the derivative with respect to the scaled time $\bar{t}$. Therefore the only parameters in the system are the scaled energies and the scaled Higgs self coupling $\bar{\lambda}$ while the lattice spacing and the gauge coupling constant do not appear explicitly.

It is easy to derive the equations of motion from the lattice Hamiltonian (2.21) and the relations (2.5) and (2.8):

$$
\begin{aligned}
\left(\dot{u}_{0}\right)_{x, i} & =\frac{1}{2} \bar{E}_{x, i}^{a} u_{x, i}^{a}, \\
\left(\dot{u}_{a}\right)_{x, i} & =-\frac{1}{2}\left[\bar{E}_{x, i}^{a}\left(u_{0}\right)_{x, i}+\epsilon^{a b c} \bar{E}_{x, i}^{b}\left(u_{c}\right)_{x, i}\right], \\
\dot{\bar{E}}_{x, i}^{a} & =i \sum_{j} \operatorname{tr}\left(\tau^{a} U_{x, i j}\right)+\bar{j}_{x, i}^{a}, \\
\ddot{\bar{\Phi}}_{x} & =-\bar{\lambda} \operatorname{tr}\left(\bar{\Phi}_{x}^{\dagger} \bar{\Phi}_{x}\right) \bar{\Phi}_{x}-6 \bar{\Phi}_{x}+\sum_{i}\left(U_{x, i} \bar{\Phi}_{x+i}+U_{x-i, i}^{\dagger} \bar{\Phi}_{x-i}\right) .
\end{aligned}
$$


Here

$$
\bar{j}_{x, i}^{a}=\frac{i}{2} \operatorname{tr}\left(\bar{\Phi}_{x}^{\dagger} \tau^{a} U_{x, i} \bar{\Phi}_{x+i}\right)
$$

is the lattice version of the gauge-covariant isospin current of the Higgs field.

In addition to satisfying these equations of motion the physical solutions must obey Gauss' law円

$$
D_{i}^{a b} E_{i}^{b}-\frac{1}{2} i g\left(\Phi^{\dagger} \tau^{a} \dot{\Phi}-\dot{\Phi}^{\dagger} \tau^{a} \Phi\right)=0
$$

with the covariant derivative $D_{i}^{a b}$ in the adjoint representation. The expression on the l.h.s. of (2.24) is a conserved quantity since it commutes with the Hamiltonian of the system. Thus to satisfy Gauss' law for all times we must only make sure that our initial conditions obey (2.24).

For a study of the thermalization properties of the different degrees of freedom the energy transfer between different sectors of the system is an important quantity. As we will see below the energy transfer from the gauge part of the system to the Higgs part is of particular interest. It is given by

$$
\frac{d}{d \bar{t}}\left(\bar{H}_{\mathrm{el}}+\bar{H}_{\mathrm{mag}}\right)=-\frac{d}{d \bar{t}}\left(\bar{H}_{\mathrm{kin}}+\bar{H}_{\mathrm{pot}}\right)=\sum_{x, i} \bar{E}_{x, i}^{a} \bar{j}_{x, i}^{a}
$$

as can be easily derived from the equations of motion. The local Higgs isospin current $\bar{j}_{x, i}^{a}$ is defined in (2.23).

\section{B. Initialization, weak coupling and continuum limit}

We initialize the system in the same way as in [6] by choosing random initial configurations:

\footnotetext{
${ }^{1}$ For brevity we present only the continuum version of Gauss' law.
} 
- The isospin directions of the Higgs fields, characterized by the angle variables $\rho_{\mathrm{H}}, \vartheta_{\mathrm{H}}$, $\varphi_{\mathrm{H}}$ are chosen randomly for each lattice site. To keep control over the initial potential energy of the Higgs fields the length of each Higgs quaternion is not randomly chosen. Instead we take for simplicity the same length $R$ for all $\Phi_{x}$ initially. Obviously the initial Higgs potential energy (2.18) increases with $R$.

- The isospin directions of the gauge fields are also randomly chosen. In this case, however, this does not mean that all the angles are arbitrary. The reason is that not only the isospin direction of the vector potential $A$ but also its amplitude enters the link variable $U$ as an angle variable. It is easy to see that $\rho_{\mathrm{G}}$ is connected to the amplitude while $\vartheta_{\mathrm{G}}$ and $\varphi_{\mathrm{G}}$ characterize the isospin direction. Therefore the latter two angles are initialized with random values on each link. To limit the initial magnetic energy we restrict the gauge field amplitude. Thus we introduce a parameter $\delta$ which controls the initial magnetic energy and choose $\rho_{\mathrm{G}}$ on each link randomly but within the range $[0,2 \pi \delta]$. For $\delta=0$ the initial magnetic energy also vanishes. For small $\delta$ the relation $H_{\text {mag }} \sim \delta^{2}$ holds.

- To satisfy Gauss' law (2.24) in an easy way we choose initially vanishing field momenta on each link, i.e. $E=0=\dot{\Phi}$. This implies that the initial electric energy and the Higgs kinetic energy, (2.15) and (2.17) respectively, vanish. Note that with non-vanishing potential but vanishing kinetic energies the system is initially in a highly off-equilibrium state.

When initialized in this way the system's further evolution is determined by the three parameters $\delta, R$, and $\bar{\lambda}$, or by the initial magnetic energy and the initial Higgs potential energy (quadratic and quartic part), respectively.

To make contact with perturbative calculations it is necessary to stay in the weak coupling regime. Furthermore, weak coupling is required for the classical calculation to be a valid approximation to the full quantum treatment. This puts constraints on the range of 
the physical parameters $\delta, R, \bar{\lambda}$. The scale invariance of the Hamiltonian allows to fix the gauge coupling at $g=1$ and the lattice spacing at $a=1$.

Weak coupling within the gauge sector is realized if the link variables are in the vicinity of unity (cf. (2.3)). In the initial state this is achieved by choosing $\delta$ small which implies that $\rho_{\mathrm{G}}$ in (2.9) is small. On the other hand, as first noted in [14] and recently worked out in [15], one encounters finite size artifacts in the pure $\mathrm{SU}(2)$ system when using too small values of $\delta$ on a finite lattice. To explore the weak gauge coupling regime one is thus restricted to a window of not too small and not too large values for $\delta$, or one must take very large lattices. With the latter option one soon runs into computer limitations.

From the Hamiltonian (2.21) one sees that weak coupling within the Higgs sector is ensured if the quadratic terms dominate the quartic one. A system with weak Higgs self coupling can thus be initialized by requiring

$$
\lambda R^{4}<R^{2}
$$

To ensure weak coupling between the gauge and the Higgs fields, the Higgs isospin current $j$ in (2.22c) should be small compared to the magnetic energy contribution? Estimating both contributions by the (maximal) field amplitudes we get the condition

$$
R^{2}<\delta
$$

The continuum limit also puts constraints on the possible parameter ranges. First, as seen from Eq. (2.3), to recover the continuum limit from the lattice formulation one needs $g a A(x) \rightarrow 0$. If the lattice simulations are supposed to yield a reasonable approximation to continuum behaviour, $\delta$ must therefore not be large. Another lattice artifact shows up if the total energy in the system is too high. Due to the compactness of the space of link variables the magnetic energy on the lattice (2.16) is limited from above by

$$
\left(\bar{H}_{\text {mag }}\right)_{\max }=4 \sum_{x, i, j}\left[1-\frac{1}{2} \operatorname{tr}(-1)\right]=24 N^{3} .
$$

\footnotetext{
${ }^{2}$ In the Abelian and continuum limits the first contribution on the r.h.s. of Eq. (2.22d) is $\nabla \times \mathbf{B}$.
} 
If the total energy in the system is much larger than this value, equipartition of the energy among the magnetic, electric and Higgs degrees of freedom may thus not be possible.

All these considerations must be taken into account when selecting the initial parameters $\delta$ and $R$ as well as the value $\bar{\lambda}$ for the Higgs self-coupling. In our calculations we have made sure to choose these parameters in such a way that we are simultaneously in the weak coupling regime and close to the continuum limit.

\section{Lyapunov exponents}

In the next Section we will study the thermalization properties of systems initialized as described above. The self-thermalization of the fields is strongly influenced by the chaoticity in its dynamics which is characterized by a set of positive Lyapunov exponents. We will here concentrate on the largest Lyapunov exponent associated with the most unstable dynamical mode. In principle one can define two such exponents, $\lambda_{\mathrm{G}}$ and $\lambda_{\mathrm{H}}$, for the gauge and Higgs fields, respectively, by using two different distance measures in the space of field configurations. The first measures the growth rate of the Euclidean distance in the gauge field sector,

$$
D_{\mathrm{G}}^{2}\left[U, U^{\prime}\right]=\sum_{x, i} \sum_{\alpha=0}^{3}\left[\left(\left(u_{\alpha}\right)_{x, i}-\left(u_{\alpha}^{\prime}\right)_{x, i}\right)^{2}+\left(\left(\dot{u}_{\alpha}\right)_{x, i}-\left(\dot{u}_{\alpha}^{\prime}\right)_{x, i}\right)^{2}\right],
$$

where $U$ and $U^{\prime}$ denote the gauge fields associated with two solutions of the equations of motion (2.22) resulting from two initially very close field configurations. The other Lyapunov exponent measures similarly the growth rate in the Higgs sector,

$$
D_{\mathrm{H}}^{2}\left[\Phi, \Phi^{\prime}\right]=\sum_{x} \sum_{\alpha=0}^{3}\left[\left(\left(\phi_{\alpha}\right)_{x}-\left(\phi_{\alpha}^{\prime}\right)_{x}\right)^{2}+\left(\left(\dot{\phi}_{\alpha}\right)_{x}-\left(\dot{\phi}_{\alpha}^{\prime}\right)_{x}\right)^{2}\right],
$$

where $\Phi$ and $\Phi^{\prime}$ are the corresponding Higgs fields from the same two solutions. As we will see in the following, the two Lyapunov exponents $\lambda_{\mathrm{G}}$ and $\lambda_{\mathrm{H}}$ turn out to be identical.

As usual the Lyapunov exponent is defined as

$$
\lambda=\lim _{t \rightarrow \infty} \lim _{D(0) \rightarrow 0} \frac{1}{t} \ln \frac{D(t)}{D(0)} .
$$


To avoid the problem of distance saturation [6] in directly measuring the Lyapunov exponent, we use the rescaling method for the evaluation of the large-time limit required in (2.31) (see [6] for more details).

In principle other definitions for the distance between field configurations are possible. In particular, one might wish to replace the Higgs distance (2.30) by a gauge invariant definition like e.g. [6,11]

$$
\sum_{x}\left|R_{x}^{2}-R_{x}^{\prime 2}\right|
$$

However, the Euclidean distances defined above are more appropriate for the rescaling method. For the pure $\mathrm{SU}(2)$ gauge system it was carefully checked that different distance measures give rise to the same Lyapunov exponent [16].

\section{THERMALIZATION PROCESSES}

In this Section we investigate various thermalization processes in the coupled SU(2) YangMills-Higgs system. We will encounter different types of thermalization processes which operate on different time scales. In particular we find that, starting with the initialization described in Sec. [I], the gauge and the Higgs subsectors each thermalize rather rapidly, while the equipartition of energy between them requires a much longer time. In the following two subsections we first present results on the fast thermalization processes associated with the gauge and the Higgs subsystems, and then discuss the system's long-time behaviour. For reasons given in Sec. \1] our study is confined to certain parameter ranges which are consistent with weak coupling and proximity to the continuum limit.

\section{A. Short-time thermalization and relaxation times}

Thermalization processes in a large system can be studied by following the evolution of the energy distribution over the microscopic degrees of freedom. In general, the distribution should approach that of a Gibbs ensemble upon equilibrium. As shown in the cases of pure 
$\mathrm{SU}(2)$ and $\mathrm{SU}(3)$ gauge fields [6], by measuring appropriate energy distributions, one can determine a parameter $T_{\mathrm{a}}$ which we call the apparent temperature henceforth. The apparent temperature $T_{\mathrm{a}}$ is related to the system's true temperature $T$, but generally differs from $T$, if the degrees of freedom of the system are not all independent, such as for a gauge theory.

We will test the degree of thermalization in various subsectors of the system by comparing the distributions for the associated energies over the lattice with a Boltzmann distribution

$$
P(\epsilon)=N_{0} f(\epsilon) \exp \left(-\epsilon / T_{\mathrm{a}}\right) .
$$

Here $\epsilon$ is the energy associated with the selected degrees of freedom (e.g. the magnetic or electric energy of the gauge fields), and $f(\epsilon)$ is an appropriate phase space factor, specified below. $N_{0}$ is a normalization constant. The distribution $P(\epsilon)$ is obtained in the simulation by sampling, at a fixed time $t$, the variable $\epsilon$ over all sites or plaquettes on the lattice; it gives the probability, averaged over the whole lattice, to find the value $\epsilon$ at a given, though arbitrary, lattice point. If this distribution has the Boltzmann shape (3.1) the system has lost all initial order in the distribution of $\epsilon$, and the entropy has reached a maximum under the constraint of fixed total energy associated with the selected degrees of freedom.

We will present measurements for four different energy distributions, the distribution $P_{\mathrm{el}}(\epsilon)$ for the electric energy $\epsilon_{\mathrm{el}}$ of the gauge fields, the distribution $P_{\mathrm{mag}}(\epsilon)$ of the magnetic energy $\epsilon_{\mathrm{mag}}$, as well as the distributions $P_{\mathrm{kin}}(\epsilon)$ and $P_{\mathrm{pot}, 2}(\epsilon)$ of the kinetic and potential energies of the Higgs field, $\epsilon_{\mathrm{kin}}$ and $\epsilon_{\mathrm{pot}, 2}$, respectively, as defined in Eqs. (2.19). In the weak coupling limit the respective phase space factors $f(\epsilon)$ are given by

$$
f(\epsilon)= \begin{cases}\epsilon^{2} & \text { for } P_{\mathrm{el}}(\epsilon), \\ \sqrt{\epsilon(8-\epsilon)} & \text { for } P_{\mathrm{mag}}(\epsilon), \\ \epsilon & \text { for } P_{\mathrm{kin}}(\epsilon), \\ \epsilon & \text { for } P_{\mathrm{pot}, 2}(\epsilon) .\end{cases}
$$

A detailed calculation of the phase space factors can be found in Appendix $A$.

By plotting $\ln [P(\epsilon) / f(\epsilon)]$ against $\epsilon$ one can read off the apparent temperature $T_{\mathrm{a}}$. It was discussed in [6, [7] that $T_{\mathrm{a}}$ is not in all cases identical with the true temperature of the system. Writing 


$$
T_{\mathrm{a}}=C(T) T
$$

one finds

$$
C= \begin{cases}1 & \text { for } P_{\mathrm{el}}(\epsilon) \\ C_{\mathrm{m}} & \text { for } P_{\mathrm{mag}}(\epsilon) \\ 1 & \text { for } P_{\mathrm{kin}}(\epsilon) \\ 1 & \text { for } P_{\mathrm{pot}, 2}(\epsilon)\end{cases}
$$

$C_{\mathrm{m}}$ is a number varying between $2 / 3$ and 1 as $T$ rises from low to high values [6]. In Ref. [6] it was argued that this can be understood in terms of the changing number of effective degrees of freedom which at low temperatures is only $2 / 3$ of that at high temperatures, due to the increasing importance of longitudinal plasma modes at high temperature. More details on the relation between the apparent temperature and the actual temperature can be found in the Appendix A.

By numerically evolving the initial configurations forward in time, we can thus follow the system's thermalization. At each moment, we can measure its energy distributions and then read off its characteristic temperatures from the measured distributions, using (3.3) for correct normalization. In Fig. 1 several characteristic temperatures of the system are plotted as functions of time, for short times below $t=6$ (in lattice units). To ensure weak coupling and closeness to the continuum limit we choose $\delta=0.2, R=0.2$, and $\lambda=1$. In this case it was found that $C_{\mathrm{m}} \approx 0.687$.

The curves in Fig. 1 demonstrate a fast thermal equilibration within the gauge field sector between the electric (solid line) and magnetic (dashed line) energy distributions. After some transient oscillations they approach, on a typical time scale of 1 lattice unit, a common temperature $T_{\mathrm{el}} \approx T_{\mathrm{mag}} \approx 0.47$. On roughly the same time scale the kinetic (dotted line) and quadratic potential (dot-dashed line) parts of the Higgs field energies also equilibrate, but at a much lower temperature $T_{\text {kin }} \approx T_{\text {pot,2 }} \approx 0.07$. This indicates that in the coupled SU(2) Higgs system the gauge and the Higgs subsector each undergo a separate, fast equilibration, starting from a highly off-equilibrium initial state. 
The approximate equality of the two associated relaxation times, $\tau_{\mathrm{G}}$ for the gauge sector and $\tau_{\mathrm{H}}$ for the Higgs sector, can be easily understood if one makes the following two hypotheses:

1. Thermalization in a chaotic system is driven by the most unstable modes and thus dominated by its largest Lyapunov exponent.

2. The gauge and the Higgs sectors are associated with the same largest Lyapunov exponent.

While it is difficult to find a rigorous proof for the first hypothesis, such a behaviour is intuitively expected. The second hypothesis can be checked in our simulation and is found to be correct (see Sec. IV).

At short times, each subsector settles down at a common temperature for all its degrees of freedom, but the temperatures of the two subsectors differ widely. This can be traced to the different initial energy content in the two subsectors. On average, the energy per degree of freedom in the gauge sector is initially 0.478, while that in the Higgs sector is only 0.060. These specific values are, of course, a result of the parameters chosen in our initialization procedure. They would be different if other parameter values were selected.

The larger total gauge energy per gauge degree of freedom compared to the total Higgs energy per Higgs degree of freedom results in the higher temperature of the gauge sector. Equilibration between the two subsectors apparently takes much longer. Since both subsectors are apparently equally "chaotic", the relative stability of the two temperatures of the subsystems indicates that energy exchange between the gauge and Higgs sectors is suppressed. This will be discussed quantitatively in the following subsection.

\section{B. Long-time equilibration and relaxation time}

As seen in Fig. 1, the coupled SU(2) Yang-Mills-Higgs system shows a very rapid thermalization within each subsector, with the relaxation times being of the order of 1 lattice unit. However, it turns out that the mutual equilibration between the two sectors is rather 
slow and requires much longer time. To study the long time behavior of the coupled system, we follow the evolution of the energies.

In Fig. 2, we plot as a function of time (on a logarithmic scale) the following energies: $E_{\mathrm{el}}\left(\right.$ solid), $E_{\mathrm{mag}}$ (dotted), $E_{\mathrm{G}}$ (short dash), $E_{\mathrm{H}}$ (long dash), and $E_{\mathrm{t}}$ (dot-dash). Here $E_{\mathrm{el}}$ is the electric energy per gauge degree of freedom:

$$
E_{\mathrm{el}}=\frac{1}{6 N^{3}} H_{\mathrm{el}}
$$

$E_{\text {mag }}$ the magnetic energy per gauge degree of freedom:

$$
E_{\mathrm{mag}}=\frac{1}{6 N^{3}} H_{\mathrm{mag}}
$$

$E_{\mathrm{H}}$ the total Higgs energy per Higgs degree of freedom:

$$
E_{\mathrm{H}}=\frac{1}{4 N^{3}}\left(H_{\mathrm{kin}}+H_{\mathrm{pot}}\right)
$$

$E_{\mathrm{G}}$ the total gauge energy per gauge degree of freedom:

$$
E_{\mathrm{G}}=E_{\mathrm{el}}+E_{\mathrm{mag}}
$$

and $E_{\mathrm{t}}$ the total energy per degree of freedom in the entire system:

$$
E_{\mathrm{t}}=\left(6 E_{\mathrm{G}}+4 E_{\mathrm{H}}\right) / 10 .
$$

$N^{3}$ is the number of lattice sites. Note that each lattice site is associated with 10 degrees of freedom: 6 gauge degrees of freedom and 4 Higgs degrees of freedom. The parameters used in the calculation are the same as in Fig. 1. In the weak coupling regime, $E_{\mathrm{H}}$ is the temperature of the Higgs sector as $E_{\mathrm{G}}$ is that of the gauge sector, while $E_{\mathrm{t}}$ will be the final temperature of the entire system after the coupled gauge and Higgs sectors reach complete equilibrium.

Again, we notice that the energy transfer from the magnetic sector to the initially unpopulated electric sector occurs on a time scale of the order of unity, which agrees with Fig. 1. The same has been seen for the Higgs kinetic energy and the Higgs potential energy 
(not shown here). However, it takes a very long time for the initially hotter gauge sector to transfer some of its energy to the cooler Higgs sector. As shown in Fig. 2, $E_{\mathrm{G}}$ (short dash) and $E_{\mathrm{H}}$ (long dash) approach the flat line $E_{\mathrm{t}} \approx 0.31$ (dot-dash) only after times of many thousand lattice units. This means that the mutual equilibration process is associated with a very long relaxation time.

Assuming an exponential relaxation law for the gauge field energy $E_{\mathrm{G}}(t)$,

$$
E_{\mathrm{G}}(t)=E_{\mathrm{G}}(\infty)+E_{0} \exp \left(-t / \tau_{\mathrm{GH}}\right)
$$

with an adjustable constant $E_{0}$, we can determine the relaxation time $\tau_{\mathrm{GH}}$ for the mutual equilibration process from a linear fit to $\ln \left[\left(E_{\mathrm{G}}(t)-E_{\mathrm{G}}(\infty)\right)\right]$. In Fig. 3 we plot $\ln \left[\left(E_{\mathrm{G}}(t)-\right.\right.$ $\left.E_{\mathrm{G}}(\infty)\right) / E_{\mathrm{G}}(\infty)$ ] as a function of time; $E_{\mathrm{G}}(\infty)$ is taken as $E_{\mathrm{t}}$ from Eq. (3.9). The solid line corresponds to the actual data while the dotted line represents the linear fit. From its slope we extract $\tau_{\mathrm{GH}}=6002 \pm 72$ lattice units.

As shown in (2.25), the rate of energy transfer from the gauge sector to the Higgs sector is given by the sum $\sum E_{x, i}^{a} j_{x, i}^{a}$. In a large system with no correlations between the signs of $E_{x, i}^{a}$ and $j_{x, i}^{a}$, this sum would be a fluctuating quantity with a zero mean value. In our calculations, the sum turns out to be fluctuating around a small negative value, which means on average there is energy flowing from the gauge sector to the Higgs sector although the flow is very slow. Furthermore, from (2.5) and (2.23), we notice that $\sum E_{x, i}^{a} j_{x, i}^{a}$ is initially proportional to $\delta$ and to $R^{2}$. Both are restricted to small values if we want to work both in the weak coupling regime and close to the continuum limit. For larger couplings (i.e. for increasing $R$ ), keeping $\delta$ small to preserve the proximity to the continuum limit, one expects faster mutual equilibration. This is borne out numerically: increasing $R$ by a factor 10 at fixed $\delta$, we found a much shorter relaxation time $\tau_{\mathrm{GH}} \approx 100$. This is, however, still much longer than the time scales for the thermalization of the gauge and the Higgs fields individually, which did not change appreciably compared to the case with the smaller value for $R$. 


\section{DETERMINATION OF THE LYAPUNOV EXPONENTS}

Our main goal in this Section is to determine from the numerical integration of the equations of motion the Lyapunov exponents associated with the gauge and the Higgs fields, and to relate them to the damping rates obtained in perturbative calculations at finite temperature [1].

Let us begin with the discussion of two technical issues. First, when determining the Lyapunov exponent from the exponential growth of the distance between two initially nearby configurations, one can in principle use different distance measures. However, for all possible choices the exponential growth in the distance will be dominated by the eigenmode associated with the largest Lyapunov exponent, which is unique to a dynamical system. Unless the chosen distance measure accidentally projects out this most unstable mode, different distance measures should thus yield the same Lyapunov exponent. (Depending on the power with which the classical fields appear in the distance measure the actually measured growth rates can be different, but they are always related to the Lyapunov exponent by a simple

multiplicative constant.) Based on these remarks one expects the two distance measures given in (2.29) and (2.30) to yield the same Lyapunov exponent.

Second, in order to relate results from numerical integration to those from perturbative calculations, we need to study the scaling behavior of the Lyapunov exponents with the energy (temperature) of the system. For the pure SU(2) gauge system the relation

$$
\lambda_{\mathrm{G}} a \approx \frac{1}{6} g^{2} E_{\mathrm{p}} a
$$

was established [6], with $E_{\mathrm{p}}=6 E_{\mathrm{G}} / 3$ denoting the gauge energy per plaquette. In [11] it was shown by a perturbative calculation with hard thermal loop resummation that, at leading order in the coupling constant, in the coupled SU(2) Yang-Mills-Higgs system the damping rate of a static gauge boson is the same as that in pure $\mathrm{SU}(2)$ gauge theory:

$$
\gamma_{\mathrm{G}}(0)=0.176 g^{2} T
$$

For the Higgs damping rate the relation 


$$
\gamma_{\mathrm{H}}(0)=0.018 g^{2} T
$$

was found [11] to hold for small values of the Higgs self coupling $\bar{\lambda}$.

Using the scaling law (4.1) together with the fact that in weak coupling the energy per plaquette $E_{\mathrm{p}}$ can be linearly related to the temperature of the system, the authors of [6.10] found that in the pure $\mathrm{SU}(2)$ and $\mathrm{SU}(3)$ gauge systems the scaled largest Lyapunov exponent coincides with twice the gauge boson damping rate. What should one expect in our case? In Ref. [11] it was speculated that here the gauge boson damping rate should be related with the Lyapunov exponent extracted from the gauge distance measure (2.29) while the Higgs boson damping rate could be connected with the Lyapunov exponent extracted from the Higgs distance measure (2.30). Since the numerical results did not support this expectation, the authors questioned the existence of a simple relationship between damping rates and Lyapunov exponents in general, raising the possibility that the coincidence found in pure $\mathrm{SU}(2)$ and $\mathrm{SU}(3)$ gauge theory was a numerical accident.

Since, however, the largest Lyapunov exponent is related to the most unstable mode in the entire coupled system and thus, as discussed above, is picked up by essentially every distance measure one can choose, we argue that one should rather expect both measures to yield the same Lyapunov exponent. We will show promptly that the numerical computations confirm this expectation. If this is the case the Lyapunov exponent can be related with at most one of the two damping rates. Since the gluon damping rate (4.2) is much larger than the Higgs damping rate (4.3) it is clear that if there is such a relation it should be between the gluon damping rate and the maximal Lyapunov exponent. To which classically computable observable the smaller Higgs damping rate can be related remains at this moment unclear. Of course, it could also be that the Lyapunov exponent is related to neither of the damping rates. To clarify this issue a careful study of the energy scaling of the Lyapunov exponent is indispensible.

In the upper part of Fig. 团we show that, within the numerical accuracy, the two distance measures (2.29) and (2.30) indeed yield the same Lyapunov exponent. (Please note the 
suppressed zero on the vertical axis.) Thus they both measure the largest Lyapunov exponent of the entire coupled system. We also see that the Lyapunov exponent shows a slight, but characteristic long-time variation which is the same for both distance measures. This behaviour will be further discussed below.

To understand the magnitude and physical meaning of the extracted Lyapunov exponent, we studied its scaling behaviour with the energy contained in the system. To this end we varied the relative energy contents in the gauge and Higgs subsectors as well as the total energy of the system, by changing $\delta$ and $R$ within the permitted ranges. We found that the Lyapunov exponent (which reflects the most unstable mode in the entire coupled system) does not scale with the total energy of the entire system, but rather with the energy stored in the gauge subsector. These scaling tests were performed on the basis of numerical runs over a few hundred to a few thousand lattice time units. On these time scales the scaling of the largest Lyapunov with the gauge field energy per plaquette was found to be accurate on the

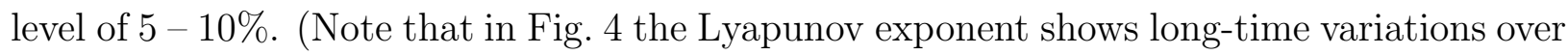
a similar range of about 10\%.) These findings indicate that the largest Lyapunov exponent is associated which the gauge field dynamics, and that the chaoticity in the gauge sector dominates the dynamics of the entire system, including the equipartition between potential and kinetic energy contributions in the Higgs subsystem.

Returning to Fig. 4 we can now discuss the magnitude of the Lyapunov exponent scaled by the energy per plaquette $E_{\mathrm{p}}$ in the gauge field sector. The lower part in Fig. $₫$ shows that for very large times of order ten thousand lattice units the Lyapunov exponent seems to converge to a constant value slightly above 0.16 . This is close to the value of about 1/6 which, according to the findings of Ref. [6], is expected (see Eq. (4.1)) if the Lyapunov exponent is related to the gluon damping rate. The observed long-time variation of the Lyapunov exponent with final convergence only after many thousand time steps is apparently connected with the already observed long-time variation of the relative energy content of the Higgs and gauge field subsectors seen in Fig. 2. As the gauge and Higgs field sectors finally reach mutual equilibrium after several ten thousand lattice time units, the scaled Lyapunov 
exponent $\lambda_{\mathrm{G}} / E_{\mathrm{p}}$ settles down to a constant asymptotic value of approximately $1 / 6$. Noting that $E_{\mathrm{p}}=2 T$ after full equilibration and inserting $g=1, a=1$, we obtain

$$
\frac{\lambda_{\mathrm{G}} a}{g^{2} T a} \approx 0.32
$$

which together with (4.2) verifies (1.3) at the level of 10\%. A more accurate verification is prohibited by the extremely slow equilibration between the gauge and Higgs subsectors in our coupled system. The numerically determined Lyapunov exponent is somewhat on the low side; according to Figs. 1 and 2, the magnitude of this discrepancy is directly related to the deviation of the gauge and Higgs field energies from equipartition.

Let us summarize the findings from this Section: We determined the gauge field Lyapunov exponent and verified that for the Yang-Mills-Higgs system it is related to the static gauge boson damping rate in the same way as for the pure gauge theory. The quantity $\lambda_{\mathrm{H}}$, as defined in (2.30) and (2.31), was shown to give the same Lyapunov exponent and therefore to be governed by the gauge degrees of freedom, too. Therefore $\lambda_{\mathrm{H}}$ cannot be related to any specific dynamics of the Higgs field, and it should not be called "Higgs Lyapunov exponent" as suggested in [11]. In particular, it is not possible to establish a relation between $\lambda_{\mathrm{H}}$ and the Higgs damping rate (4.3). Unfortunately, we have not been able to come up with an alternative observable in the dynamics of classical fields which could be related to $\gamma_{\mathrm{H}}$.

\section{FURTHER DISCUSSION}

We close this paper with a discussion of our results in comparison with the recent work presented in [11]. For small values of the Higgs self coupling $\bar{\lambda}$, Biró and Thoma [11] found that the Lyapunov exponents $\lambda_{\mathrm{H}}$ and $\lambda_{\mathrm{G}}$ deduced from the Higgs and gauge fields, respectively, agree within the numerical accuracy. For very large values of the Higgs self coupling, on the other hand, $\lambda_{\mathrm{H}}$ was found to decrease, thereby breaking the coincidence between $\lambda_{\mathrm{H}}$ and $\lambda_{\mathrm{G}}$. For the case of small $\bar{\lambda}$ we have verified the coincidence of $\lambda_{\mathrm{H}}$ and $\lambda_{\mathrm{G}}$. It has a natural explanation since, as we already discussed, in principle any distance measure 
between two neighboring trajectories in phase space simply yields the maximal Lyapunov exponent of the entire system.

In view of this argument, the disagreement between $\lambda_{\mathrm{H}}$ and $\lambda_{\mathrm{G}}$ at large $\bar{\lambda}$ at first seems surprising. If, however, the distance measure projects out the eigenmode with the largest Lyapunov exponent, it can yield a different value for the growth rate. This is apparently what happens at very large values of $\bar{\lambda}$ for the Higgs distance (2.32) used in [11]: As already pointed out in [11], for large $\bar{\lambda}$ the dynamics of the Higgs amplitude (2.12) becomes completely determined by the quartic self-interaction term (2.19e), thereby effectively freezing the Higgs field amplitude $R$. Thus this degree of freedom starts to decouple from the rest of the system, and it may happen that the growth of perturbations in the Higgs amplitude is no longer influenced by the maximal Lyapunov exponent associated with the gauge field, at least, over the time scale used for the determination of the Lyapunov exponent.

We have not studied here the case of large $\bar{\lambda}$, for the following reasons: First, if one increases $\bar{\lambda}$ without decreasing the Higgs amplitude one leaves the perturbative regime (2.26). Then the computed Lyapunov exponents can no longer be compared with the damping rates from hard thermal loop calculations. Second, for very large $\bar{\lambda}$ the energy density of the quartic interaction term (2.198) and thus also the total energy density become very large. As discussed in Sec. IIB, in this case one runs into lattice artifacts due to the compactness of the magnetic energy on the lattice.

While we agree with Biró and Thoma [11] about the coincidence of $\lambda_{\mathrm{G}}$ and $\lambda_{\mathrm{H}}$, we disagree with their determination of the temperature. Consequently we also differ in the interpretation of their results with respect to their connection with perturbative damping rates, believing that their rather negative conclusions are not justified. Biró and Thoma [11] use the Stefan-Boltzmann law for 10 degrees of freedom

$$
\frac{E_{\mathrm{tot}}}{V}=\frac{\pi^{2}}{3} k_{\mathrm{B}} T\left(\frac{k_{\mathrm{B}} T}{\hbar c}\right)^{3}
$$

to extract the temperature $T$ from the total energy density $E_{\text {tot }} / V$ of the system. Here we put in $k_{\mathrm{B}}, \hbar$, and $c$ explicitly (elsewhere we take $k_{\mathrm{B}}=\hbar=c=1$ ). As can be seen from 
the occurrence of Planck's constant in (5.1), this law makes explicit use of the quantized nature of excitation energies in a gas of (massless) particles. As such it can not be applied to a classical field theory. The classical Yang-Mills-Higgs fields on the lattice correspond to a dynamical system of coupled anharmonic oscillators which in thermal equilibrium and for weak coupling obeys the relation

$$
\frac{E_{\mathrm{tot}}}{N^{3} a^{3}}=\frac{10 T}{a^{3}}+o(g, \lambda)
$$

Thus the energy per degree of freedom is directly proportional to the temperature, and not to its fourth power.

Even more crucial is our finding that the whole system equilibrates only after a very long time. Since the authors of [1] did not use the rescaling method for extracting the Lyapunov exponents, they ran very soon into the problem of distance saturation due to the compactness of the gauge group [6], and thus their analysis was necessarily restricted to times which were short compared to the mutual equilibration time between the gauge and Higgs sectors. If the Lyapunov exponent is determined on such short time scales, the total energy of the system does not tell anything about the temperature of the relevant gauge subsector no matter whether it is determined from (5.1) or (5.2). For this reason Biró and Thoma missed the specific connection of the Lyapunov exponent with the gauge subsystem rather than with the entire coupled Yang-Mills-Higgs system. Only an analysis like the one presented here can uncover the fact that after very short thermalization times one can cleanly define separate temperatures for the gauge and the Higgs subsystems, and that the maximal Lyapunov exponent actually scales with the temperature of the gauge sector already long before global equilibration between the gauge and Higgs sectors sets in.

In conclusion, we have studied the high temperature behavior of the coupled YangMills-Higgs system in the weak coupling regime close to the continuum limit. We found that the coupled system undergoes thermalization in stages: while the gauge and Higgs subsectors self-thermalize very rapidly, equipartition of the energy between these two sectors takes several orders of magnitude longer. We expect and have qualitative evidence for a 
connection between the rapid thermalization time scale within each of the two subsectors and the magnitude of the largest Lyapunov exponent; to establish this relation quantitatively requires, however, a much more detailed and computer-intensive investigation. Since we found that the largest Lyapunov exponent is associated with the gauge field and scales with the gauge field energy, we conclude that thermalization of both the gauge and Higgs subsectors is driven by the chaotic dynamics of classical non-Abelian gauge fields. The Higgs subsector thus thermalizes via its coupling to the chaotic gauge fields (gluon heat bath) and not due its own intrinsic non-linearity via the selfcoupling $\bar{\lambda}$. The scaling of the Lyapunov exponent with the gauge field energy was used to verify that, even in the presence of a coupling to matter fields, the largest Lyapunov exponent still agrees with twice the damping rate of a static gauge boson.

The analytical results of Thoma and Biró [11] from thermal perturbation theory show that in the coupled Yang-Mills-Higgs system also the Higgs damping rate is proportional to $g^{2} T$ and thus, from the general arguments given e.g. in [10], is expected to survive in the classical limit. At the moment it remains an open question whether it is possible to identify an observable in the dynamical evolution of classical Yang-Mills-Higgs fields which can be associated with the Higgs boson damping rate in a similar way as the largest Lyapunov exponent is related to the gluon damping rate.

\section{ACKNOWLEDGMENTS}

The authors thank M. Thoma and T. Biró for fruitful discussions. This work was supported in part by the U.S. Department of Energy (Grant No. DE-FG02-96ER40945) and

by the North Carolina Supercomputing Center. U.H. and S.L. acknowledge support by the Deutsche Forschungsgemeinschaft (DFG) and the Bundesministerium für Bildung, Wissenschaft, Forschung und Technologie (BMBF). 


\section{APPENDIX A: CALCULATION OF PHASE SPACE FACTORS}

In this Appendix we derive the phase space factors presented in Sec. IIIA. In general it is difficult to calculate the energy distribution over the microscopic degrees of freedom of an arbitrary system evolving in time. It is equivalent to solving the equations of motion. However, as shown in the main part of the paper, the gauge and Higgs sectors of the system equilibrate separately very rapidly (albeit reaching different temperatures). In addition we are working in a regime where weak coupling is assumed between the gauge and Higgs sectors as well as within each sector. This significantly simplifies the task of calculating the energy distributions of different degrees of freedom.

To determine how the electric energy is distributed over the lattice sites for the thermalized gauge sector at temperature $T_{\mathrm{G}}$, we neglect its coupling to the Higgs fields and write the partition function for the gauge sector as

$$
Z=\int \prod_{x, i, a} d \bar{E}_{x, i}^{a} d \mu\left(U_{x, i}\right) \exp \left[-\frac{\bar{H}_{\mathrm{el}}+\bar{H}_{\mathrm{mag}}}{T_{\mathrm{G}}}\right] \delta\left(D_{x, i}^{a b} \bar{E}_{x, i}^{b}\right) \delta\left(F\left(U_{x, i}\right)\right),
$$

where the first $\delta$-function takes into account the constraints imposed by Gauss' law, and the second one represents gauge fixing of the form $F\left(U_{x, i}\right)=0$, which involves link variables only. $\mu(U)$ is the Haar measure defined on the SU(2) group mainfold.

For the electric energy on a site, we define its distribution function $P_{\mathrm{el}}(\epsilon)$ as

$$
\begin{aligned}
P_{\mathrm{el}}(\epsilon)= & Z^{-1} \int \prod_{x, i, a} d \bar{E}_{x, i}^{a} d \mu\left(U_{x, i}\right) \exp \left[-\frac{\bar{H}_{\mathrm{el}}+\bar{H}_{\mathrm{mag}}}{T_{\mathrm{G}}}\right] \\
& \times \delta\left(D_{x, i}^{a b} \bar{E}_{x, i}^{b}\right) \delta\left(F\left(U_{x, i}\right)\right) \delta\left(\epsilon-\frac{1}{2} \sum_{i, a} \bar{E}_{x_{0}, i}^{a} \bar{E}_{x_{0}, i}^{a}\right) \\
= & Z^{-1} \int \prod_{x, i, a} d \bar{E}_{x, i}^{a} d \mu\left(U_{x, i}\right) d \lambda_{x}^{a} d \kappa \delta\left(F\left(U_{x, i}\right)\right) \\
& \times \exp \left[-\frac{\bar{H}_{\mathrm{el}}+\bar{H}_{\mathrm{mag}}}{T_{\mathrm{G}}}+i \sum_{x, i, a, b} \lambda_{x}^{a} D_{x, i}^{a b} \bar{E}_{x, i}^{b}+i \kappa\left(\epsilon-\frac{1}{2} \sum_{i, a} \bar{E}_{x_{0}, i}^{a} \bar{E}_{x_{0}, i}^{a}\right)\right]
\end{aligned}
$$

where we have introduced Lagrange multipliers $\lambda_{x}^{a}$ and $\kappa$ to account for the $\delta$-functions. The electric and magnetic energies are defined in (2.15) and (2.16), respectively. Since the

electric fields appear only up to quadratic order in (A2), the integrations over $\bar{E}_{x, i}^{a}$ can be performed with the result 


$$
\begin{aligned}
P_{\mathrm{el}}(\epsilon) \sim & \int \prod_{x, i, a} d \mu\left(U_{x, i}\right) d \lambda_{x}^{a} d \kappa \delta\left(F\left(U_{x, i}\right)\right) \operatorname{det}^{-1 / 2}\left[\left(\frac{1}{T_{\mathrm{G}}}+i \kappa \delta_{x, x_{0}}\right) \delta_{x, x^{\prime}} \delta_{i, i^{\prime}} \delta_{a, a^{\prime}}\right] \\
& \times \exp \left[-\frac{\bar{H}_{\mathrm{mag}}}{T_{\mathrm{G}}}-\frac{T_{\mathrm{G}}}{2} \sum_{x, i, a, b}^{x \neq x_{0}}\left(D_{x, i}^{a b} \lambda_{x}^{a}\right)^{2}-\frac{T_{\mathrm{G}}}{2\left(1+i \kappa T_{\mathrm{G}}\right)} \sum_{i, a, b}\left(D_{x_{0}, i}^{a b} \lambda_{x_{0}}^{a}\right)^{2}+i \kappa \epsilon\right] \\
\sim & \int \prod_{x, i, a} d \mu\left(U_{x, i}\right) d \lambda_{x}^{a} d \kappa \delta\left(F\left(U_{x, i}\right)\right)\left(1+i \kappa T_{\mathrm{G}}\right)^{-9 / 2} \\
& \times \exp \left[-\frac{\bar{H}_{\mathrm{mag}}}{T_{\mathrm{G}}}-\frac{T_{\mathrm{G}}}{2} \sum_{x, i, a, b} \frac{\left(D_{x, i}^{a b} \lambda_{x}^{a}\right)^{2}}{1+i \kappa T_{\mathrm{G}} \delta_{x, x_{0}}}+i \kappa \epsilon\right] .
\end{aligned}
$$

The integration over the Lagrange multipliers $\lambda_{x}^{a}$ can be evaluated similarly. After that, the whole integral factorizes into integrations over the link variables and the Lagrange multiplier $\kappa$. The integration over the link variables is $\epsilon$-independent and can be factored out as a constant. We thus obtain

$$
\begin{aligned}
P_{\mathrm{el}}(\epsilon) & \sim \int_{-\infty}^{+\infty} d \kappa\left(1+i \kappa T_{\mathrm{G}}\right)^{-3} \exp (i \kappa \epsilon) \\
& \sim \frac{\epsilon^{2}}{T_{\mathrm{G}}^{3}} \exp \left(-\epsilon / T_{\mathrm{G}}\right)
\end{aligned}
$$

which yields the electric phase space factor $f_{\mathrm{el}}(\epsilon)=\epsilon^{2}$. Three points should be stressed here: First, the above result is exact for a pure $\mathrm{SU}(2)$ gauge system and approximately valid for the weakly coupled SU(2) Higgs system. Second, the apparent temperature agrees with the true temperature, i.e. $C_{\mathrm{el}}=1$. Finally we mention that $\epsilon^{2}$ is replaced by $\epsilon^{N^{2}-2}$ for the general case of $\mathrm{SU}(N)$.

In deriving the distribution for the magnetic energy on a plaquette, we again neglect the coupling to the Higgs fields and write

$$
\begin{aligned}
P_{\mathrm{mag}}(\epsilon)= & Z^{-1} \int \prod_{x, i, a} d \bar{E}_{x, i}^{a} d \mu\left(U_{x, i}\right) \exp \left[-\frac{\bar{H}_{\mathrm{el}}+\bar{H}_{\mathrm{mag}}}{T_{\mathrm{G}}}\right] \\
& \times \delta\left(D_{x, i}^{a b} \bar{E}_{x, i}^{b}\right) \delta\left(F\left(U_{x, i}\right)\right) \delta\left(\epsilon-4\left(1-\frac{1}{2} \operatorname{tr} U_{x_{0}, i_{0} j_{0}}\right)\right) .
\end{aligned}
$$

Assuming weak coupling within the gauge sector itself, we neglect the nonlinear term in the Gauss' law, which reduces $\mathbf{D} \cdot \mathbf{E}=0$ to a linear form: $\nabla \cdot \mathbf{E}=0$. Then the integration over the electric fields decouples from that over the link variables and can be factored out as a constant independent of $\epsilon$. Hence we find 


$$
P_{\mathrm{mag}}(\epsilon) \sim \int \prod_{x, i} d \mu\left(U_{x, i}\right) \exp \left(-\bar{H}_{\mathrm{mag}} / T_{\mathrm{G}}\right) \delta\left(F\left(U_{x, i}\right)\right) \delta\left(\epsilon-4\left(1-\frac{1}{2} \operatorname{tr} U_{x_{0}, i_{0} j_{0}}\right)\right)
$$

Now the integration is over the link variables $U_{x, i}$, while the energy is expressed in terms of the plaquettes $U_{x, i j}$. In order to carry out the integration, we use a technique described in [18 to transform the integral over link variables into an integral over plaquettes [17]:

$$
P_{\mathrm{mag}}(\epsilon) \sim \int \prod_{x, i, j} d \mu\left(U_{x, i j}\right) \exp \left(-\bar{H}_{\mathrm{mag}} / T_{\mathrm{G}}\right) \prod_{c} \delta\left(U_{c}-1\right) \delta\left(\epsilon-4\left(1-\frac{1}{2} \operatorname{tr} U_{x_{0}, i_{0} j_{0}}\right)\right)
$$

where the $\delta$-function $\delta\left(U_{c}-1\right)$ imposes a constraint on each elementary cube $c$. These constraints are the lattice version of the Bianchi identities, which correspond to $\mathbf{D} \cdot \mathbf{B}=$ 0 in the continuum limit. $U_{c}$ is an $\mathrm{SU}(2)$ matrix defined in terms of the link variables associated with the cube $c$. The transformation from links to plaquettes corresponds to the transformation from vector potentials to magnetic field strengths in the continuum limit. For details on the transformation and the exact form of $U_{c}$, we refer the reader to [18 and [17]. Now we consider (A7) in two limiting cases: $T_{\mathrm{G}} \rightarrow 0$ and $T_{\mathrm{G}} \rightarrow \infty$.

In the low temperature limit, i.e. when the field amplitudes are small, the non-Abelian part of the constraints becomes negligible and hence the non-Abelian Bianchi identities $\mathbf{D} \cdot \mathbf{B}=0$ reduce to their Abelian equivalents $\nabla \cdot \mathbf{B}=0$. On the lattice, they take the form of $\sum_{i j \in c} B_{i j}=0$, where the sum runs through the six plaquettes forming the surface of a cube. These are strong constraints which completely eliminate the longitudinal components of the $B$ fields and reduce the effective number of degrees of freedom by $1 / 3$. So in this limit, the apparent temperature $T_{\mathrm{a}}=2 T_{\mathrm{G}} / 3$.

In the high temperature limit, we expand the constraints in terms of the irreducible representations of the group $\mathrm{SU}(2)$ [13]:

$$
\delta\left(U_{c}-1\right)=\sum_{J}(2 J+1) \chi_{J}\left(U_{c}\right), \quad J=0, \frac{1}{2}, 1, \ldots
$$

where $\chi_{J}\left(U_{c}\right)$ is the trace of $U_{c}$ in the $(2 J+1)$-dimensional representation. For $T_{\mathrm{G}} \rightarrow \infty$, the dominant contribution to (A7) comes from $J=0$. Taking $J=0$ and neglecting those with $J \neq 0$ is equivalent to ignoring the constraints imposed by the Bianchi identities and treating 
the plaquettes as independent variables. Then it is not surprising to find that the apparent temperature $T_{\mathrm{a}}$ obtained in measuring $P_{\mathrm{mag}}(\epsilon)$ coincides with the actual temperature $T_{\mathrm{G}}$.

While it is easy to do the integrations in the limiting cases, it is difficult to calculate A7) for intermediate temperatures. Noting that the major effect of the constraints $\delta\left(U_{c}-1\right)$ on the distribution function is to change its logarithmic slope from the actual temperature $T_{\mathrm{G}}$ to the apparent temperature $T_{\mathrm{a}}$, we assume that these constraints can be effectively taken into account by replacing $T_{\mathrm{G}}$ by $T_{\mathrm{a}}$ in (A7) and write

$$
P_{\mathrm{mag}}(\epsilon) \sim \int \prod_{x, i, j} d \mu\left(U_{x, i j}\right) \exp \left(-\bar{H}_{\mathrm{mag}} / T_{\mathrm{a}}\right) \delta\left(\epsilon-4\left(1-\frac{1}{2} \operatorname{tr} U_{x_{0}, i_{0} j_{0}}\right)\right)
$$

Of course, the validity of this assumption is to be justified and the relation between $T_{\mathrm{a}}$ and $T_{\mathrm{G}}$ to be established by numerical calculations.

The evaluation of (A9) is straightforward. Making use of the quaternion representation $U=u^{0}+i \tau^{a} u^{a}$ for a plaquette $U$ and the explicit form of the Haar measure on the $\mathrm{SU}(2)$ group manifold

$$
d \mu(U)=\frac{1}{\pi^{2}} \delta\left(\frac{1}{2} \operatorname{tr}\left(U^{\dagger} U\right)-1\right) d u^{0} d u^{1} d u^{2} d u^{3}
$$

one can easily carry out the integration in (A9) and obtain

$$
P_{\mathrm{mag}}(\epsilon) \sim f_{\mathrm{mag}}(\epsilon) \exp \left(-\epsilon / T_{\mathrm{a}}\right)
$$

where $f_{\text {mag }}(\epsilon)=\sqrt{\epsilon(8-\epsilon)}$. The relation $T_{\mathrm{a}}=C\left(T_{\mathrm{G}}\right) T_{\mathrm{G}}$ can be determined numerically and $C\left(T_{\mathrm{G}}\right)$ varies from $2 / 3$ to 1 as $T_{\mathrm{G}}$ increases from zero to infinity [6].

Note that, for the sake of convenience, we have calculated the distribution of the electric energy on a site and that of the magnetic energy on a plaquette. Hence the apparent electric and magnetic distributions do not coincide even at low temperature where, in principle, one can treat the electric and magnetic energy in exactly the same way and arrive at the same distributions. However, as seen in Fig. 1, the different results for $T_{\mathrm{a}}$ relate to the same value of $T_{\mathrm{G}}$ in the electric and magnetic sector. 
To derive how the Higgs kinetic and potential energies are distributed over the lattice sites for the thermalized Higgs sector at temperature $T_{\mathrm{H}}$, we neglect both the gauge-Higgs coupling and the Higgs self-coupling. Then it is straightforward to derive

$$
P_{\text {kin,pot }}(\epsilon) \sim \frac{\epsilon}{T_{\mathrm{H}}^{2}} \exp \left(-\epsilon / T_{\mathrm{H}}\right)
$$

from which we obtain $f_{\text {kin }}(\epsilon)=f_{\text {pot }, 2}(\epsilon)=\epsilon$.

In principle, perturbative corrections to the results obtained in (A4), (A11), and (A12) can be calculated. For our case at hand, however, it turns out that the formulae derived above are in good agreement with the numerical results. This is not surprising because our numerical calculations are performed for weak coupling. 


\section{REFERENCES}

* Permanent address: Institut für Theoretische Physik, Universität Regensburg, D-93040 Regensburg, Germany

+ Also at Yerevan Physics Institute, Yerevan, Armenia

[1] C. DeTar, in Quark-Gluon Plasma 2, edited by R.C. Hwa, (World Scientific, Singapore, 1995), p. 1.

[2] E. Braaten and R. D. Pisarski, Phys. Rev. D42, 2156 (1990).

[3] M.H. Thoma, in Quark-Gluon Plasma 2, edited by R.C. Hwa, (World Scientific, Singapore, 1995), p. 51.

[4] E. Braaten and A. Nieto, Phys. Rev. D53, 3421 (1996).

[5] T.S. Biró, S. Matinyan, and B. Müller, Chaos and Gauge Field Theory (World Scientific, Singapore, 1994).

[6] T.S. Biró, C. Gong, B. Müller, and A. Trayanov, Int. J. Mod. Phys. C5, 113 (1994).

[7] V.P. Silin, Zh. Eksp. Teor. Fiz. 38, 1577 (1960) [Sov. Phys. JETP 11, 1136 (1960)].

[8] U. Heinz, Ann. Phys. (N.Y.) 168, 148 (1986).

[9] P.F. Kelly, Q. Liu, C. Lucchesi, C. Manuel, Phys. Rev. D50, 4209 (1994).

[10] T.S. Biró, C. Gong, and B. Müller, Phys. Rev. D52, 1260 (1995).

[11] T.S. Biró and M.H. Thoma, preprint hep-ph/9603339, to appear in Phys. Rev. D.

[12] K. Wilson, Phys. Rev. D10, 2445 (1974).

[13] M. Creutz, Quarks, Gluons, and Lattices (Cambridge University Press, Cambridge, 1983).

[14] B. Müller and A. Trayanov, Phys. Rev. Lett. 68, 3387 (1992). 
[15] B. Müller, Duke University preprint DUKE-TH-96-118 (chao-dyn/9607001).

[16] C. Gong, Phys.Rev. D49, 2642 (1994).

[17] C. Gong, Ph.D. thesis, Duke University, 1994 (unpublished).

[18] G.G. Batrouni, Nucl. Phys. B208, 467 (1982). 


\section{FIGURES}

FIG. 1. Short-time equilibration inside the gauge and the Higgs subsectors. Four characteristic temperatures are plotted as functions of time: the temperature of the electric gauge sector $T_{\mathrm{el}}$ (solid line), the temperature of the magnetic gauge sector $T_{\text {mag }}$ (dashed line), the temperature of the kinetic Higgs sector $T_{\text {kin }}$ (dotted line), and the temperature of the quadratic part of the potential Higgs sector $T_{\text {pot,2 }}$ (dot-dashed line). Parameters: $\delta=0.2, R=0.2, g=1, \lambda=1$, and $a=1$.

FIG. 2. Long-time equilibration between the gauge and the Higgs subsectors. Five energies are plotted as functions of time: the electric energy per gauge field degree of freedom $E_{\mathrm{el}}$ (solid line), the magnetic energy per gauge field degree of freedom $E_{\text {mag }}$ (dotted line), the total gauge field energy $E_{\mathrm{G}}=E_{\mathrm{el}}+E_{\text {mag }}$ per gauge field degree of freedom (short dash), the Higgs field energy per Higgs field degree of freedom $E_{\mathrm{H}}$ (long dash), and the total energy per degree of freedom $E_{\mathrm{t}}$ (dot-dashed line). Parameters as in Fig. 1.

FIG. 3. Determination of the relaxation time $\tau_{\mathrm{GH}}$ associated with the long-time equilibration between the gauge and the Higgs subsectors. The solid represents the numerical data while the dotted line is a linear fit to the data.

FIG. 4. Upper graph: coincidence of $\lambda_{\mathrm{G}}$ and $\lambda_{\mathrm{H}}$. Lower graph: convergence of the gauge field Lyapunov exponent. $E_{\mathrm{p}}$ is the gauge field energy per plaquette. The fields were rescaled after each period $\Delta t=30$. 


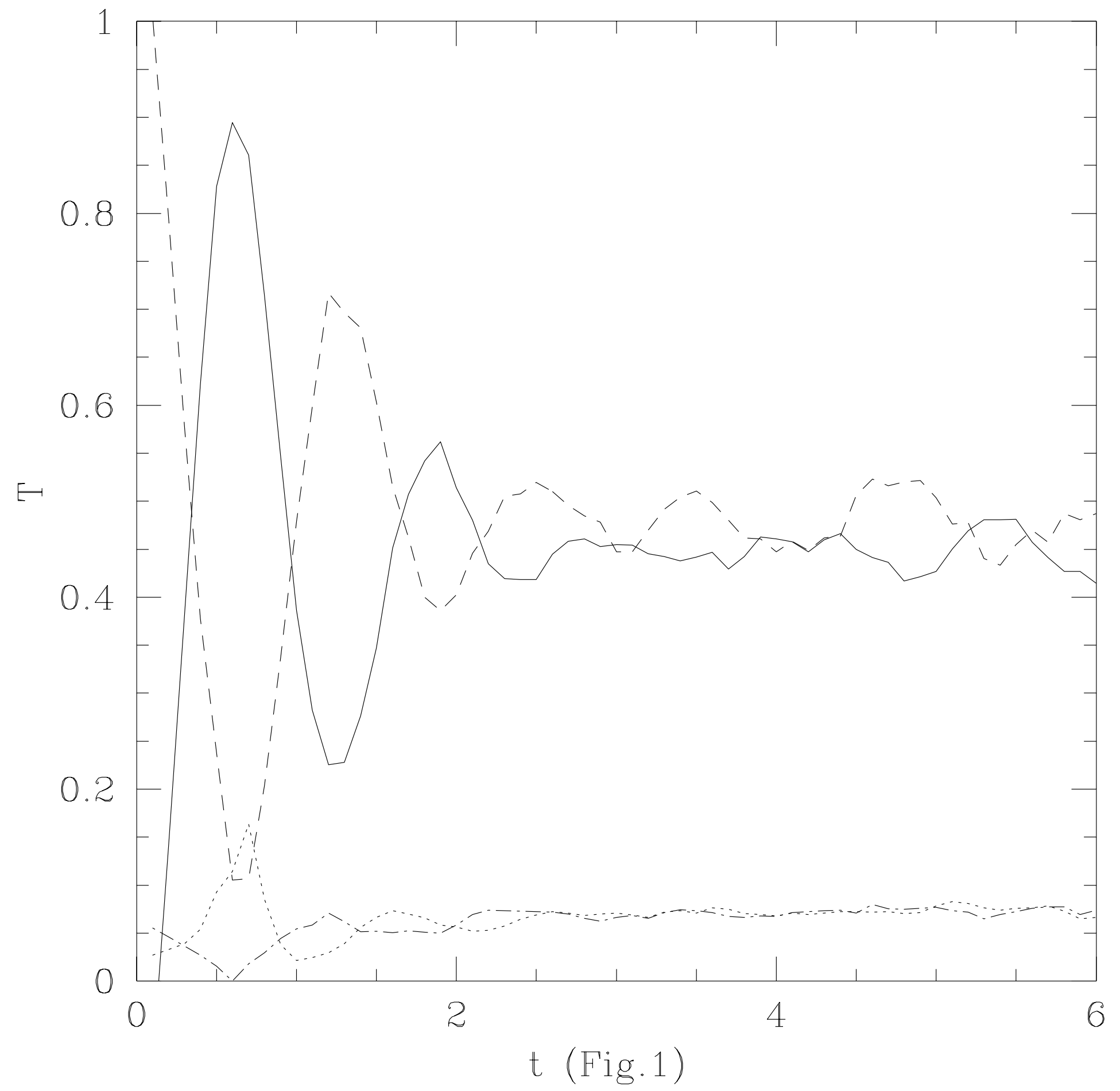




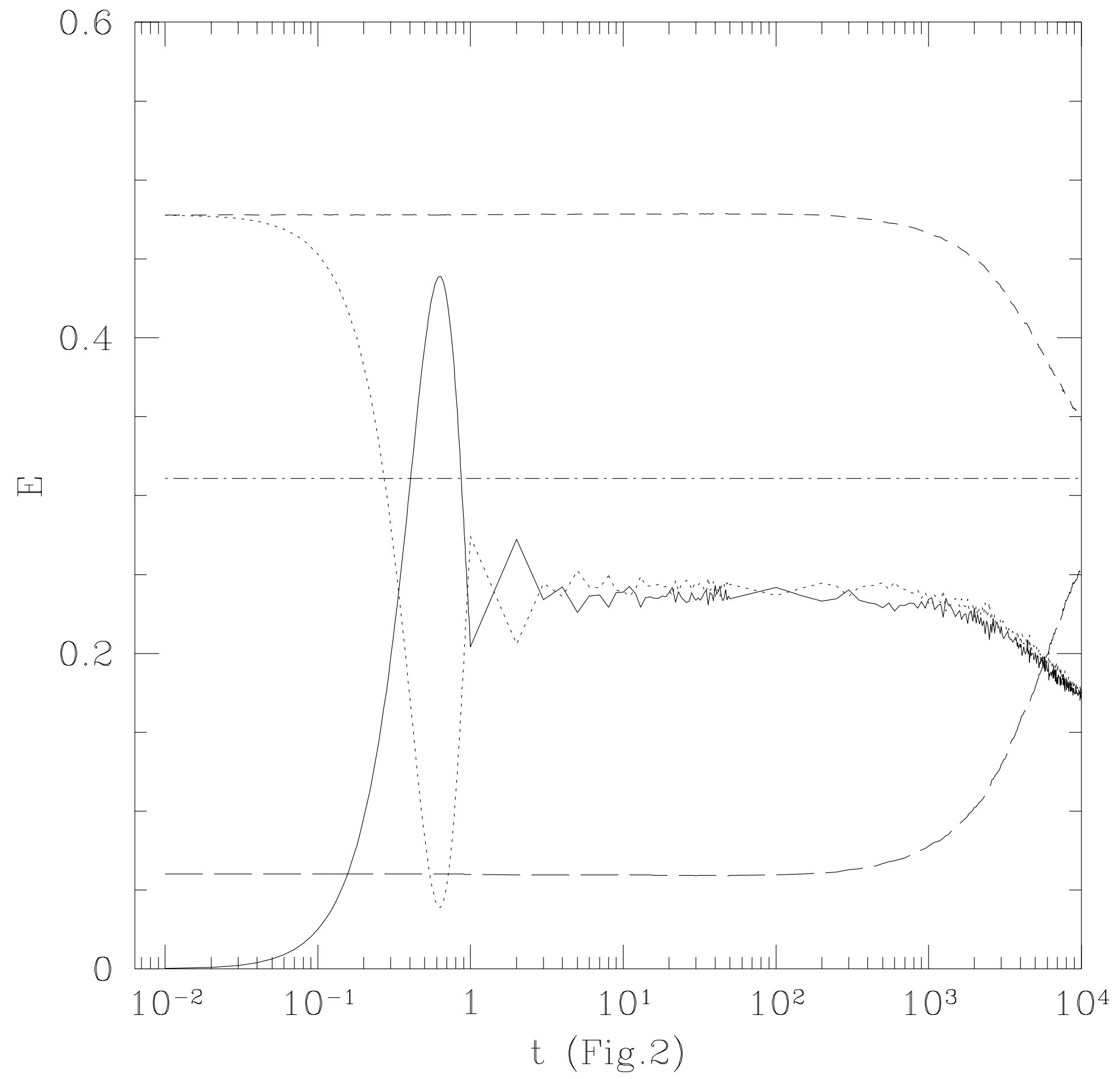




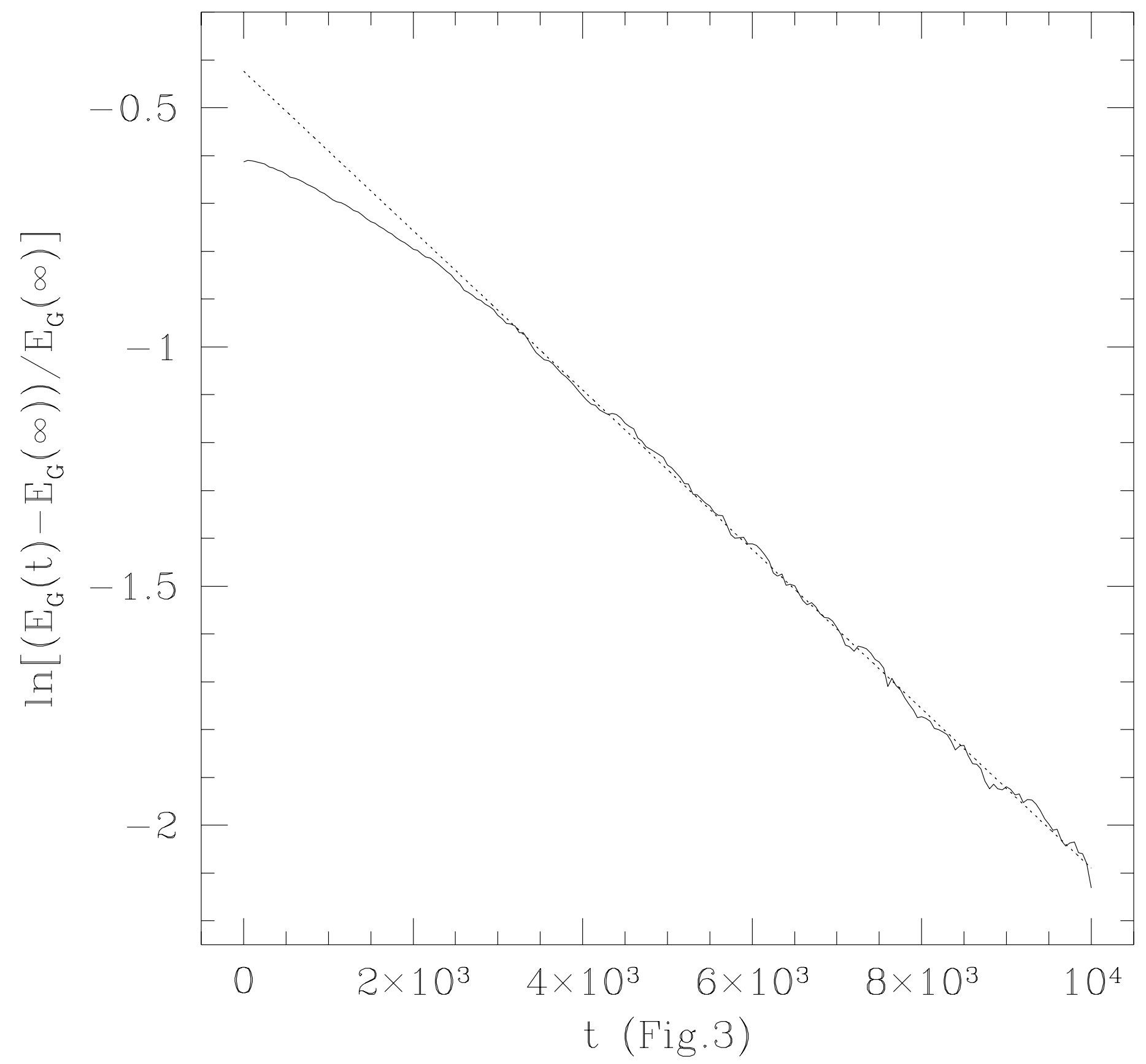



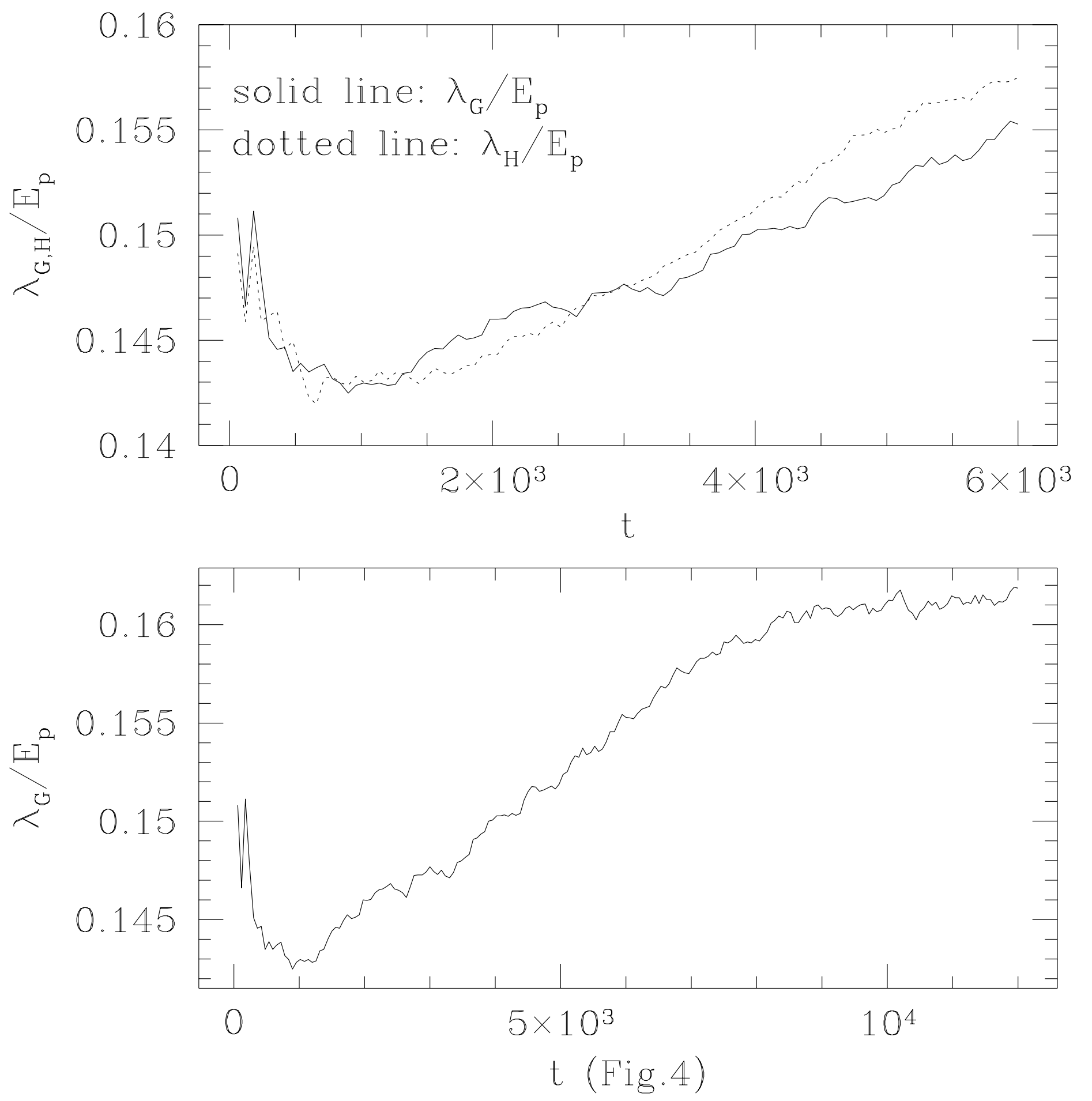